\title{
Matrix stiffness-upregulated LOXL2 promotes fibronectin production, MMP9 and CXCL12 expression and BMDCs recruitment to assist pre-metastatic niche formation
}

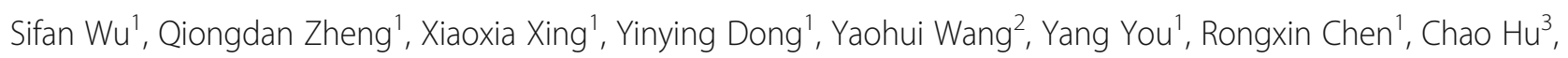
Jie Chen', Dongmei Gao', Yan Zhao', Zhiming Wang ${ }^{4}$, Tongchun Xue' ${ }^{1}$ Zhenggang Ren ${ }^{1}$ and Jiefeng Cui ${ }^{*}$

\begin{abstract}
Background: Higher matrix stiffness affects biological behavior of tumor cells, regulates tumor-associated gene/ miRNA expression and stemness characteristic, and contributes to tumor invasion and metastasis. However, the linkage between higher matrix stiffness and pre-metastatic niche in hepatocellular carcinoma (HCC) is still largely unknown.
\end{abstract}

Methods: We comparatively analyzed the expressions of LOX family members in HCC cells grown on different stiffness substrates, and speculated that the secreted LOXL2 may mediate the linkage between higher matrix stiffness and premetastatic niche. Subsequently, we investigated the underlying molecular mechanism by which matrix stiffness induced LOXL2 expression in HCC cells, and explored the effects of LOXL2 on pre-metastatic niche formation, such as BMCs recruitment, fibronectin production, MMPs and CXCL12 expression, cell adhesion, etc.

Results: Higher matrix stiffness significantly upregulated LOXL2 expression in HCC cells, and activated JNK/C-JUN signaling pathway. Knockdown of integrin $\beta 1$ and a5 suppressed LOXL2 expression and reversed the activation of above signaling pathway. Additionally, JNK inhibitor attenuated the expressions of p-JNK, p-c-JUN, c-JUN and LOXL2, and shRNA-C-JUN also decreased LOXL2 expression. CM-LV-LOXL2-OE and rhLOXL2 upregulated MMP9 expression and fibronectin production obviously in lung fibroblasts. Moreover, activation of Akt pathway contributed to LOXL2-induced fibronectin upregulation. LOXL2 in CM as chemoattractant increased motility and invasion of BMCs, implicating a significant role of LOXL2 in BMCs recruitment. Except that, CM-LV-LOXL2-OE as chemoattractant also increased the number of migrated HCC cells, and improved chemokine CXCL12 expression in lung fibroblasts. The number of HCC cells adhered to surface of lung fibroblasts treated with CM-LV-LOXL2-OE was remarkably higher than that of the control cells. These results indicated that the secreted LOXL2 facilitated the motility of HCC cells and strengthened CTCS settlement on the remodeled matrix "soil".

Conclusion: Integrin $\beta 1 / a 5 / J N K / C-J U N$ signaling pathway participates in higher matrix stiffness-induced LOXL2 upregulation in HCC cells. The secreted LOXL2 promotes fibronectin production, MMP9 and CXCL12 expression and BMDCs recruitment to assist pre-metastatic niche formation.

Keywords: Hepatocellular carcinoma, Matrix stiffness, LOXL2, Pre-metastatic niche

\footnotetext{
* Correspondence: cui.jiefeng@zs-hospital.sh.cn

${ }^{1}$ Liver Cancer Institute, Zhongshan Hospital, Fudan University and Key

Laboratory of Carcinogenesis and Cancer Invasion, Ministry of Education, 136

Yi Xue Yuan Road, Shanghai 200032, People's Republic of China

Full list of author information is available at the end of the article
}

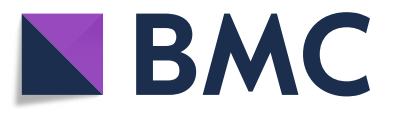

(c) The Author(s). 2018 Open Access This article is distributed under the terms of the Creative Commons Attribution 4.0 International License (http://creativecommons.org/licenses/by/4.0/), which permits unrestricted use, distribution, and

reproduction in any medium, provided you give appropriate credit to the original author(s) and the source, provide a link to the Creative Commons license, and indicate if changes were made. The Creative Commons Public Domain Dedication waiver (http://creativecommons.org/publicdomain/zero/1.0/) applies to the data made available in this article, unless otherwise stated. 


\section{Background}

Increasing evidences suggest that matrix stiffness influences biological behaviors of cells such as cell proliferation [1], differentiation [2, 3], migration [4], and metabolism [5], regulates disease-associated genes/miRNA expression [6-9], stemness [10], chemoresistance [11], and contributes to tumor invasion and metastasis [12]. Hepatocellular carcinoma (HCC) is one of the most frequent tumor in China and the third leading cause of cancer-related mortality worldwide [13]. Over $80 \%$ of HCC patients have cirrhosis or advanced fibrosis background. The mortality rate of $\mathrm{HCC}$ with cirrhosis background rises in some developed countries [14]. Currently, higher liver stiffness has become a strong predictor in clinic for HCC development and prognosis $[1,15]$. Our previous studies have demonstrated that increased matrix stiffness not only upregulates VEGF and OPN expressions in HCC cells [16, 17], but also strengthens their stemness characteristics [10]. Other literatures also support that increased matrix stiffness elevates the expression of integrin $\beta 1$, and is positively correlated with the invasion and metastasis of HCC patients with cirrhosis [12]. Additionally, higher matrix stiffness can alter chemotherapeutic responses of HCC cells [1]. The formation of tumor pre-metastatic niche, which occurs in the distant target organ/tissue, is a critical molecule event at the late stage of tumor metastasis, and determines the implementation of distant metastasis. Generally, pre-metastatic niche resembles as the fertile "soil" and assists circulating tumor cells settlement in target organ/tissue and facilitates tumor distant metastasis [18]. In these years, the identified molecules and cells in distant metastasis tissue of different tumor animal models including the primary tumor-derived soluble factors, vesicles, exosome and bone marrow derived cells (BMDCs), etc. gradually confirmed the existence of pre-metastasis niche in the most types of malignant tumors $[18,19]$. However, little is known about the linkage between matrix stiffness and pre-metastatic niche in HCC. Lysyl oxidase (LOX) family is composed of LOX, LOXL1, LOXL2, LOXL3 and LOXL4. All of these five members have highly conserved C-terminal domain that contains copper binding motif, lysine tryosylquinone residues and a cytokine receptor-like domain, therefore they exhibit similar catalytic activity [20]. However, their amino-terminal regions are different, determine their different roles in protein-protein interaction [21] To date, only few soluble factors such as tumor secreted LOXL2 [22], exosomes [23] exhibit important pathological roles in formation of pre-metastatic niche in HCC metastasis. FoxM1b stimulates the expressions of LOX and LOXL2 to induce pre-metastatic niche formation in the lung of HCC animal model [24]. Although higher matrix stiffness forces the expression of LOX in HCC as described previously [16], it remains largely unknown that which member of LOX family in HCC plays dominating function in matrix stiffness-induced effects on pre-metastatic niche. In the study, we investigated the expression of LOX family members in HCC cells grown on different substrate stiffness and its regulation mechanism, and subsequently analyzed possible biological effects of LOXL2 on pre-metastatic niche formation such as BMDCs recruitment, fibronectin production, MMPs and CXCL12 expression,cell adhesion, etc.

\section{Methods}

A gel-based culture system with tunable substrate stiffness

A gel-based culture system with tunable substrate stiffness was established as our previous study [16].

\section{Cells and cell culture}

$\mathrm{MHCC} 97 \mathrm{H}$, a highly metastatic type of $\mathrm{HCC}$ cell, was established at the Liver Cancer Institute of Fudan University. A lowly metastatic HCC cell Hep3B and human embryo lung fibroblast (HELF) were obtained from the Cell Bank of Shanghai Institute of Biochemistry and Cell Biology, CAS. Approximately $1 \times 10^{6} \mathrm{HCC}$ cells in $0.3 \mathrm{ml}$ of medium were spread evenly onto COL1-coated gel substrate with tunable stiffness in dish and cultured for $2 \mathrm{~h}$ at room temperature. Subsequently, $12 \mathrm{ml}$ medium was added into dish and the cells were cultured in incubator for $48 \mathrm{~h}$. MHCC97H cells and HELF cells were all grown in Dulbecco's Modified Eagle's Medium (DMEM, Gibco) supplemented with $10 \%$ fetal bovine serum (FBS; Biowest) and $1 \%$ penicillin/streptomycin (Gibco), and Hep3B cells in minimum essential medium (MEM, Gibco) with $10 \%$ fetal FBS and $1 \%$ penicillin/streptomycin.

\section{Collection of conditioned medium (CM) from HCC cells}

HCC cells transfected with LV-scramble (NC) or LVLOXL2-OE were cultured in serum-free DMEM for $24 \mathrm{~h}$, and their culture supernatants were collected and then sterilized by a $0.2 \mu \mathrm{m}$ filter (Millipore, Schwalbach, Germany). The collected $\mathrm{CM}$ were stored at $-80{ }^{\circ} \mathrm{C}$ for further use.

\section{Recombinant plasmid construction of c-JUN and transient transfection}

The shRNA sequence (CcggcgGACCTTATGGCTA CAGTAAcg CTGTAGCCATAAGGTCCGTTTTTg) targeting human c-JUN gene was designed by the Shanghai GeneChem, Co. Ltd., China. The uppercase letters and lowercase letters represented c-JUN-specific sequence and hairpin sequences, respectively. The double-strand DNA was cloned into GV248 vector and recombinant plasmid expressing c-JUN-shRNA was constructed. HCC cells were transfected with recombinant plasmid shRNA-c-JUN using lipofectamine 2000, and the transfected HCC cells were collected after 48 h culture. 


\section{Western blot}

Total proteins of the collected cells were extracted in lysis buffer containing RIPA buffer (Beyotime, China), $1 \mathrm{mM}$ phenylmethanesulfonyl fluoride (Beyotime, China), and 10\% PhosSTOP (Roche, Switzerland). Approximately $20 \mu \mathrm{g}$ total protein was loaded and separated by $12 \%$ SDSPAGE, then transferred onto a polyvinylidene difluoride membrane (Millipore, USA). Subsequently, the PVDF membrane was blocked in TBS/Tween with $5 \%$ fat-free milk and incubated with the following primary antibodies: GAPDH (1:1000, Cell Signal Technology), LOX (1:1000, Abcam), LOXL1 (1:1000, Abcam), LOXL2 (1:1000, Abcam; 1:2000, Novus Biologicals), LOXL3 (1:100, Santa Cruz), LOXL4 (1:500, Abnova), c-JUN (1:1000, Proteintech), p-c-JUN (Ser73) (1:1000, Cell Signal Technology), JNK, p-JNK (Thr183/Tyr185) (1:1000, Cell Signal Technology), MMP2 (1:1000, Abcam), MMP9 (1:1000, Cell Signal Technology). The membrane was further incubated with HRP-conjugated secondary antibody (1:4000, Proteintech). Finally, the target protein band was visualized using an electrochemiluminescence kit (Thermo).

\section{Quantitative reverse transcription polymerase chain reaction (qRT-PCR)}

Total RNA was extracted from HCC cells using Trizol reagent (Invitrogen). mRNA was reverse-transcribed into cDNA using a RevertAid/First Strand Synthesis Kit (Thermo Scientific), and then cDNA template was further amplified with gene specific primer and SYBR Green PCR Master Mix kit (Invitrogen). Three replicates were set for each primer in each group, the relative mRNA expression was normalized to GAPDH and reported as $2^{-\Delta \Delta C t}$. The primer sequences of the detected genes are listed in Table 1.

Stable knockdown expression of integrin $\beta 1$, integrin a5 and overexpression of LOXL2 in HCC cells using lentiviralmediated RNAi/overexpression technology

The interference sequences targeting human integrin $\beta 1$ (ITG $\beta 1,5$ '-CCTCCAGATGACATAGAAA-3'), integrin $\alpha 5$
(ITGA5, 5'-TCAGGAACGAGTCAGAATT-3'), and human LOXL2 overexpression sequence (see Additional file 1) were designed by the Shanghai GeneChem, Co. Ltd., China. Target sequence to integrin $\beta 1$ or $\alpha 5$ genes was cloned into the plasmid pGCSIL, and LOXL2 overexpression sequence was cloned into GV416 vector. LV-integrin-shRNA and LV-LOXL2-OE were obtained by co-transfecting above plasmids and packing plasmids Helper1.0 and Helper2.0 into $293 \mathrm{~T}$ cells. The viral supernatant (at multiplicity of infection $=10)$ containing Eni.S and $5 \mu \mathrm{g} / \mathrm{ml}$ polybrene was added into HCC cells. After $12 \mathrm{~h}$, the transfection solution was replaced with normal culture medium for continuing culture to obtain stably-transfected HCC cells. Assessment of transfection efficiency see Additional file 2: Figure S1.

\section{Immunohistochemistry analysis}

The detailed procedure of immunohistochemistry was described in our previous work [16]. Cell slides were incubated with primary antibody against LOXL2 (1:400, Abcam), c-JUN (1:50, Proteintech) at $4{ }^{\circ} \mathrm{C}$ overnight, and then reacted with secondary antibody conjugated with HRP (1:200, DingguoBio, Beijing). Staining intensity and the percentage of the stained tissues were scored by two independent observers. Photographs of four representative sites were captured under high-power magnification $(\times 200)$ by the Leica QWin Plus v3 software with identical setting parameters. The density of positive staining was measured by Image-Pro Plus v6.2 software (Media Cybernetics Inc., USA). Integrated optical density of the positive stains in each photograph was measured, and its ratio to the total area of each photograph was calculated.

\section{JNK inhibition assay}

JNK-IN-8 (MedChem Express, USA) is a selective JNK1/ $2 / 3$ inhibitor, and inhibits phosphorylation of downstream molecule c-JUN. JNK-IN-8 dissolved in DMSO was diluted into $1 \mu \mathrm{M}$ work solution with complete culture medium, and the same amount of DMSO was set as the control $\left(\mathrm{V}_{\mathrm{DMSO}}: \mathrm{V}\right.$ (complete culture medium) $\left.=0.5 \%\right)$. HCC

Table 1 Primer sequence used for qRT-PCR

\begin{tabular}{lll}
\hline Gene symbol & Forward primer (5'-3') & Reverse primer (5'-3') \\
\hline LOX & TTACCCAGCCGACCAAGATA & CCTTCAGCCACTCTCCTCTG \\
LOXL1 & TACGATGTGCGGGTGCTAC & ATGCTGTGGTAATGCTGGTG \\
LOXL2 & AGGATGTCGGTGTGTGTG & TTGCGGTAGGTTGAGAGGAT \\
LOXL3 & TGGAGTTCTATCGTGCCAATGA & CCTGAGGCTTCGACTGTTGT \\
LOXL4 & ACTGTAGGCTGCTGGGACAC & GGTTCACAATCACCTGGAAGA \\
MMP2 & GTTCATTTGGCGGACTGT & AGGGTGCTGGCTGAGTAG \\
MMP9 & GGGACGCAGACATCGTCATC & TCGTCATCGTCGAAATGGGC \\
CXCL12 & GTTCAAAGCCAGCGTC & TAGTTCACCCCAAAGGA \\
GAPDH & CTCCTCCACCTTGACGC & CCACCACCCTGTTGCTGT \\
\hline
\end{tabular}


cells grown on high stiffness substrates were treated with JNK-IN-8 for $48 \mathrm{~h}$.

\section{Cell motility assay}

Approximately $4 \times 10^{5} \mathrm{HCC}$ cells suspended in $200 \mu \mathrm{l}$ serum-free DMEM were placed into the upper chamber of a Bodyen Chamber (Millipore), $250 \mu \mathrm{l}$ DMEM containing 20\% FBS and $250 \mu \mathrm{l} \mathrm{CM-LV-LOXL2-OE} \mathrm{were}$ mixed and placed in lower chamber. After $48 \mathrm{~h}$ incubation, the migrating cells in the membrane were fixed with $4 \%$ paraformaldehyde for $30 \mathrm{~min}$, and then stained in crystal violet for $30 \mathrm{~min}$. The number of cells in six random fields were counted under a light microscope.

\section{BMCs isolation and BMCs recruitment assay in vitro}

The cells in femurs and tibias of mice were harvested by PBS flushing, and then treated with Red Blood Cell Lysis Buffer (Beyotime Biotechnology) to isolate bone marrow cells (BMCs). For BMCs Invasion assay, Transwell inserts (Millipore) were coated with Matrigel (BD Biosciences) at $37^{\circ} \mathrm{C}$ for $2 \mathrm{~h} .1 \times 10^{6}$ fresh BMCs suspended in $200 \mu \mathrm{l}$ serum-free DMEM were seeded into the upper chamber. $500 \mu \mathrm{l}$ of mixed medium as a chemoattractant $(250 \mu \mathrm{l}$ DMEM with $10 \%$ FBS and $250 \mu \mathrm{l} \mathrm{CM-LV-}$ LOXL2-OE) were placed in lower chamber. After $24 \mathrm{~h}$ incubation, cells that invaded through the membrane were founded in the lower chamber suspension and counted. The cell motility assay was performed in a similar method, except that the cells were seeded into the uncoated inserts.

\section{Cell adhesion assay}

After HELF cells grew and reached 70\% confluence in 6 well plate, they were treated with CM-LV-LOXL2-OE. When the treated HELF cells were close to $100 \%$ confluence, $2 \times 10^{5}$ GFP-labeled HCC cells were put upon the treated HELF cells. Twenty-four hours later, the attached MHCC $97 \mathrm{H}$ cells on the surface of HELF cells were photographed using a fluorescence microscopy (Leica). The number of attached HCC cells was measured by counting six random microscope fields $(\times 200)$, and data were expressed as a means \pm SEM.

\section{Statistical analysis}

Data analysis was performed using SPSS 20.0 statistical software (SPSS Inc., Chicago, IL). All data were expressed as mean \pm standard error of the mean (SEM). Statistical analysis was performed by Student's t-test. $p<0.05$ indicating statistical significance. All experiments have been performed in triplicate.

\section{Results}

The expression levels of LOX family members in HCC cells grown on different stiffness substrates

We used $6 \mathrm{KPa}, 10 \mathrm{KPa}$, and $16 \mathrm{KPa}$, as our previous report [16], to represent the stiffness values of normal liver tissue, fibrosis, and cirrhosis tissue. We first detected mRNA and protein expressions of LOX family members in HCC cells growing on different stiffness substrates, and found that the expressions of LOX, LOXL1, LOXL2 and LOXL4 except for LOXL3 were all significantly upregulated in MHCC97H and Hep3B cells under higher stiffness stimulation (Fig. 1a, b). LOXL3 expression was not detected at the protein level. According to the expression levels of LOX family members in HCC cells under different stiffness stimulation and the significance role of LOX family members in premetastatic niche $[22,25]$, we speculated that the secreted LOXL2 from HCC cells might mediate the linkage between higher matrix stiffness and pre-metastatic niche, and facilitate tumor distant metastasis. In addition, higher matrix stiffness stimulation significantly altered shape and spread area of HCC cells. MHCC97H and Hep3B cells grown on 10, $16 \mathrm{KPa}$ stiffness substrate exhibited an extension and expansion morphology, different from small round shape on $6 \mathrm{KPa}$ stiffness substrate (Fig. 2a). Morphological alteration perhaps means a great change in their malignant characteristics. To confirm LOXL2 expression level in vivo, we further applied a HCC tissue microarray established previously [16] to analyze the effect of liver matrix stiffness on LOXL2 expression. Our data showed that LOXL2 highly expressed in HCC tissues with high and medium liver stiffness backgrounds as compared with that of HCC tissue with normal liver stiffness background (Fig. 2b (i)), in agreement with the results in vitro.

\section{Integrin $\beta 1 / \alpha 5 / J N K / c-J U N$ signaling pathway may be involved in matrix stiffness-induced LOXL2 upregulation in HCC cells}

Higher matrix stiffness upregulated the expression of LOXL2 in MHCC97H and Hep3B cells, simultaneously significantly improved the phosphorylation levels of JNK, the phosphorylation and expression levels of c-JUN (Fig. 3a), illustrating that high matrix stiffness might activate the JNK/ c-JUN signaling pathway. We further analyzed the expression of signal molecule of c-JUN in HCC tissues, and found that the expression level of c-JUN elevated as liver stiffness background increased. (Fig. 2b (ii)). Given that JNK/c-JUN pathway is associated with LOX expression [26, 27], we conjectured that activation of JNK/c-JUN pathway might participate in high matrix stiffness-induced LOXL2 upregulation. Previously, we reported that integrin $\beta 1$ and $\alpha 5$ as major bridge molecules delivered stiffness signal into HCC cells [16]. Here, knockdown of integrin $\beta 1$ or integrin $\alpha 5$ 

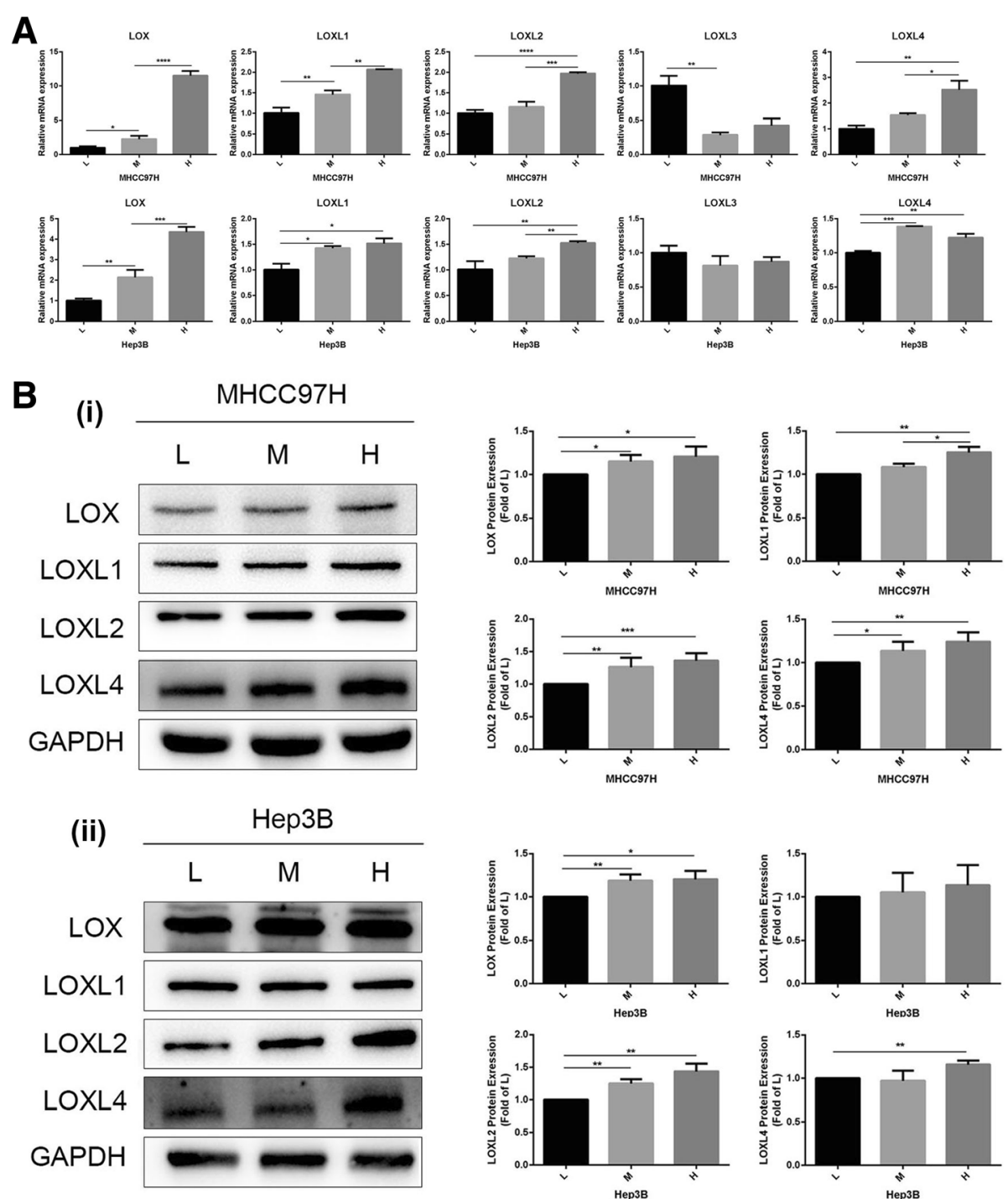

Fig. 1 Expressions of the LOX family members in HCC cells under diverse stiffness stimulation. (a) mRNA expression levels of LOX, LOXL1, LOXL2, LOXL3 and LOXL4 in MHCC97H and Hep3B cells grown on $6 \mathrm{KPa}(\mathrm{L}), 10 \mathrm{KPa}(\mathrm{M})$, and $16 \mathrm{KPa}(\mathrm{H})$ stiffness substrate. (b) (i, ii) The protein expression level of LOX, LOXL1, LOXL2, LOXL4 in MHCC97H and Hep3B cells grown on $6 \mathrm{KPa}(\mathrm{L}), 10 \mathrm{KPa}(\mathrm{M})$, and $16 \mathrm{KPa}(\mathrm{H})$ stiffness substrate. LOXL3 was not detected at the protein level. Error bar represents SEM, ${ }^{*} p<0.05,{ }^{* *} p<0.01$ and ${ }^{* * *} p<0.001$

suppressed the phosphorylation levels of JNK and c-JUN obviously in HCC cells grown on higher stiffness substrate, also downregulated the expression of LOXL2 and c-JUN (Fig. 3b), demonstrating that higher matrix stiffnessactivated JNK/c-JUN pathway was partially reversed. Thereby, activation of integrin $\beta 1 / \alpha 5$-JNK/c-JUN pathway is required for higher matrix stiffness-induced LOXL2 upregulation in HCC cells.

\section{JNK inhibitor and shRNA-c-JUN remarkably suppress} LOXL2 expression and JNK/C-JUN pathway activation in HCC cells grown on high stiffness substrate

JNK-IN-8, a selective JNK inhibitor, inhibits phosphorylation of its downstream molecule c-JUN [28]. We used the JNK inhibitor to treat HCC cells under higher stiffness stimulation and further measured LOXL2 expression and the activation of JNK/C-JUN pathway. As shown in Fig. 4a, JNK inhibitor sharply attenuated the expression of p-JNK, p-c-JUN, c-JUN as well as LOXL2 in HCC cells grown on higher stiffness substrate, and shRNA-c-JUN also decreased LOXL2 expression remarkably (Fig. 4b). These above results strongly support that JNK/C-JUN pathway specifically modulates matrix stiffness-induced LOXL2 upregulation. Simultaneously, a co-inhibitor of JNK and integrin $\beta$ showed a more pronounced decrease in phosphorylation levels of JNK and c-JUN and expression of LOXL2 as compared with JNK inhibitor or integrin $\beta 1$ alone (Fig. $4 \mathrm{~b}$ ), further confirming that integrin $\beta 1$ and 


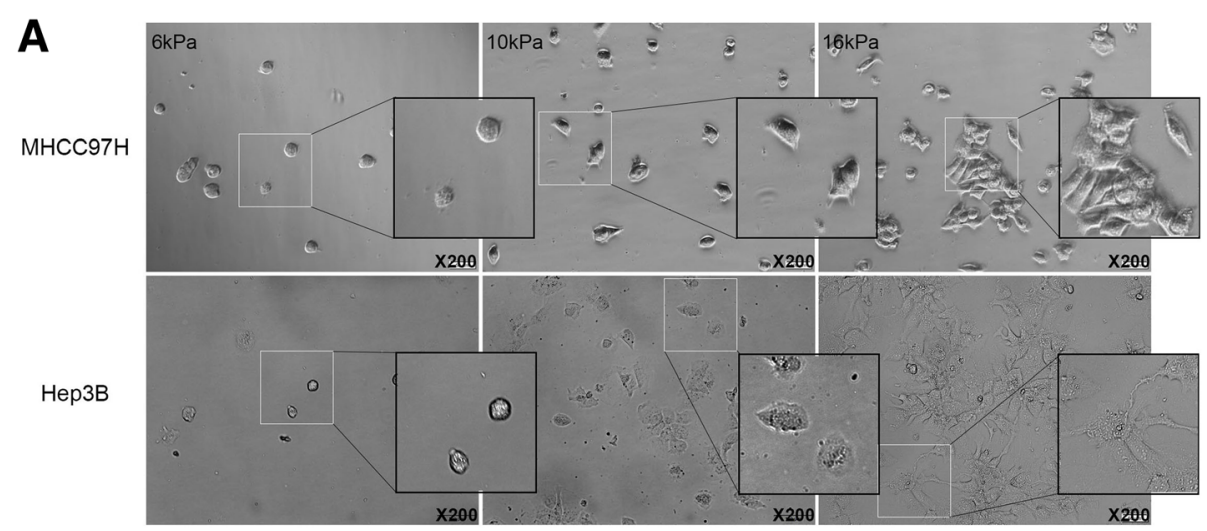

B (i)
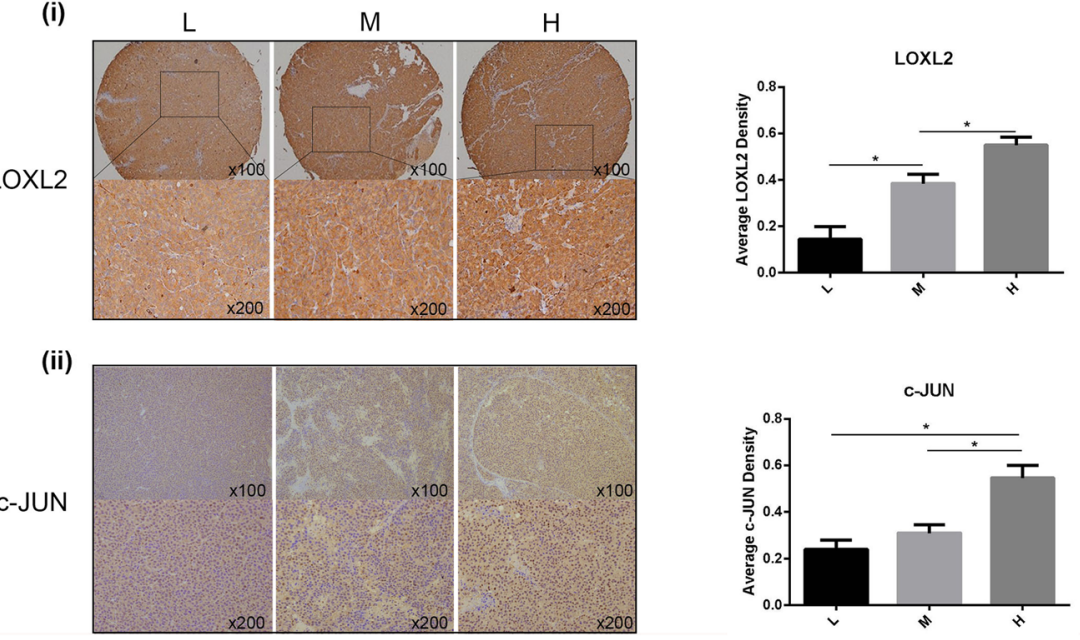

Fig. 2 Morphological changes of HCC cells under different stiffness stimulation and the expression of LOXL2 and c-JUN in HCC tissue with different liver stiffness backgrounds. (a) Morphology of MHCC97H and Hep3B cells cultured on 6 kPa, $10 \mathrm{kPa}$, and $16 \mathrm{kPa}$ stiffness substrates. (b) (i) The expression levels of LOXL2 in HCC tissues with normal liver stiffness backgrounds (groups L), medium liver stiffness backgrounds (group M) and high liver stiffness backgrounds (group H). (ii) The expression levels of c-JUN in HCC tissues with different liver stiffness backgrounds. Error bar represents $\mathrm{SEM},{ }^{*} p<0.05$

JNK/c-JUN pathway contributed to matrix stiffnessinduced LOXL2 upregulation. Taken together, we concluded that high matrix stiffness indeed activated the JNK/c-JUN pathway in HCC cells and then upregulated LOXL2 expression.

\section{LOXL2 increases motility and invasion of BMCs,} simultaneously improves MMP9 and CXCL12 expression, as well as fibronectin production in lung fibroblasts, which assists HCC cells settlement on them

BMDCs recruitment and matrix remodeling are two important characteristics of pre-metastatic niche formation. We assessed the effects of LOXL2 on BMDCs recruitment and matrix remodeling in vitro by BMCs migration/invasion assay and MMP expression. As shown in Fig. 5a, the number of migrated BMCs or invaded BMCs in CM-LVLOXL2-OE group was significantly higher than that of CM-NC group. LOXL2 in CM as chemoattractant increased motility and invasion of BMCs, implicating a significant role of LOXL2 in BMCs recruitment. We analyzed
MMP9 and MMP2 expression in lung fibroblasts treated with CM-LV-LOXL2-OE and found that CM-LV-LOXL2OE significantly elevated MMP9 expression, but had no effect on MMP2 expression (Fig. 5b). A recombinant protein LOXL2 (rhLOXL2) also showed same effects on MMP9 and MMP2 expressions, suggesting that the secreted LOXL2 upregulates MMP9 expression in lung fibroblasts and is in favor of matrix remodeling in lung tissue. On the other hand, CM-LV-LOXL2-OE remarkably upregulated fibronectin expression in lung fibroblasts, simultaneously it increased phosphorylation levels of AKT (Ser473 and Thr308) significantly (Fig. 5c (i, ii)). Furthermore, rhLOXL2 with the concentration of $20 \mathrm{nM}$ and $50 \mathrm{nM}$ obviously elevated phosphorylation levels of AKT and fibronectin expression in lung fibroblasts (Fig. 5c (i, iii)), demonstrating that AKT pathway may participate in LOXL2induced fibronectin upregulation. Undoubtedly, both MMP9 upregulation and matrix protein deposition created a suitable "soil" in lung for circulating tumor cells colonization and growth. Fibronectin is a glycoprotein of 

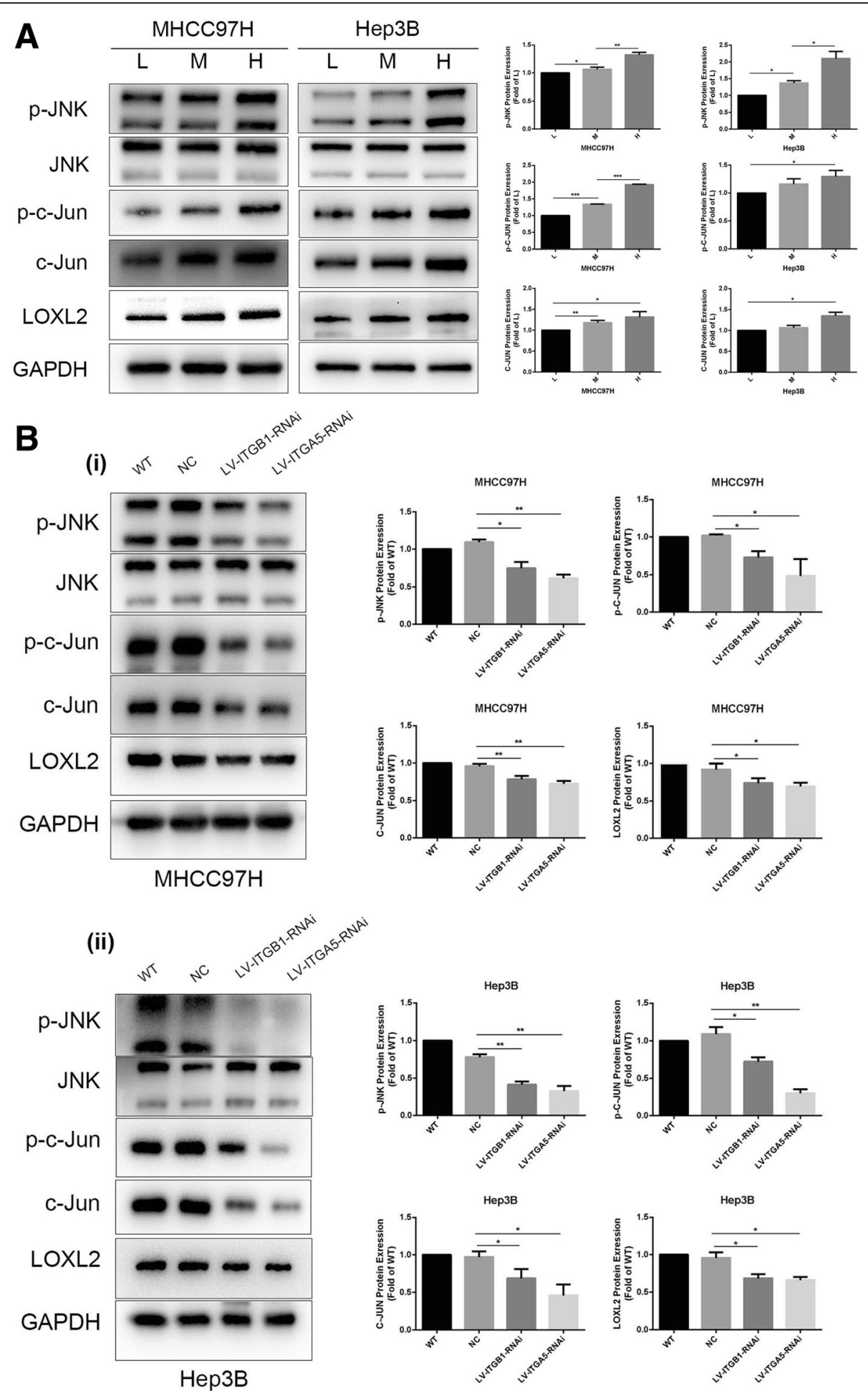

Fig. 3 Higher matrix stiffness upregulated LOXL2 expression in HCC cells via activating integrin $\beta 1 / a 5 / J N K / C-J U N$ signaling pathway. (a) Increased matrix stiffness upregulated LOXL2 expression and activated JNK/c-JUN signaling pathway in MHCC97H and Hep3B cells. (b) (i, ii) Knockdown of integrin $\beta 1$ or integrin a5 obviously suppressed the expression levels of p-JNK, p-c-JUN, c-JUN and LOXL2 in HCC cells grown on 16 KPa stiffness substrate. Error bar represents SEM, ${ }^{*} p<0.05,{ }^{* *} p<0.01$ and ${ }^{* * *} p<0.001$ 


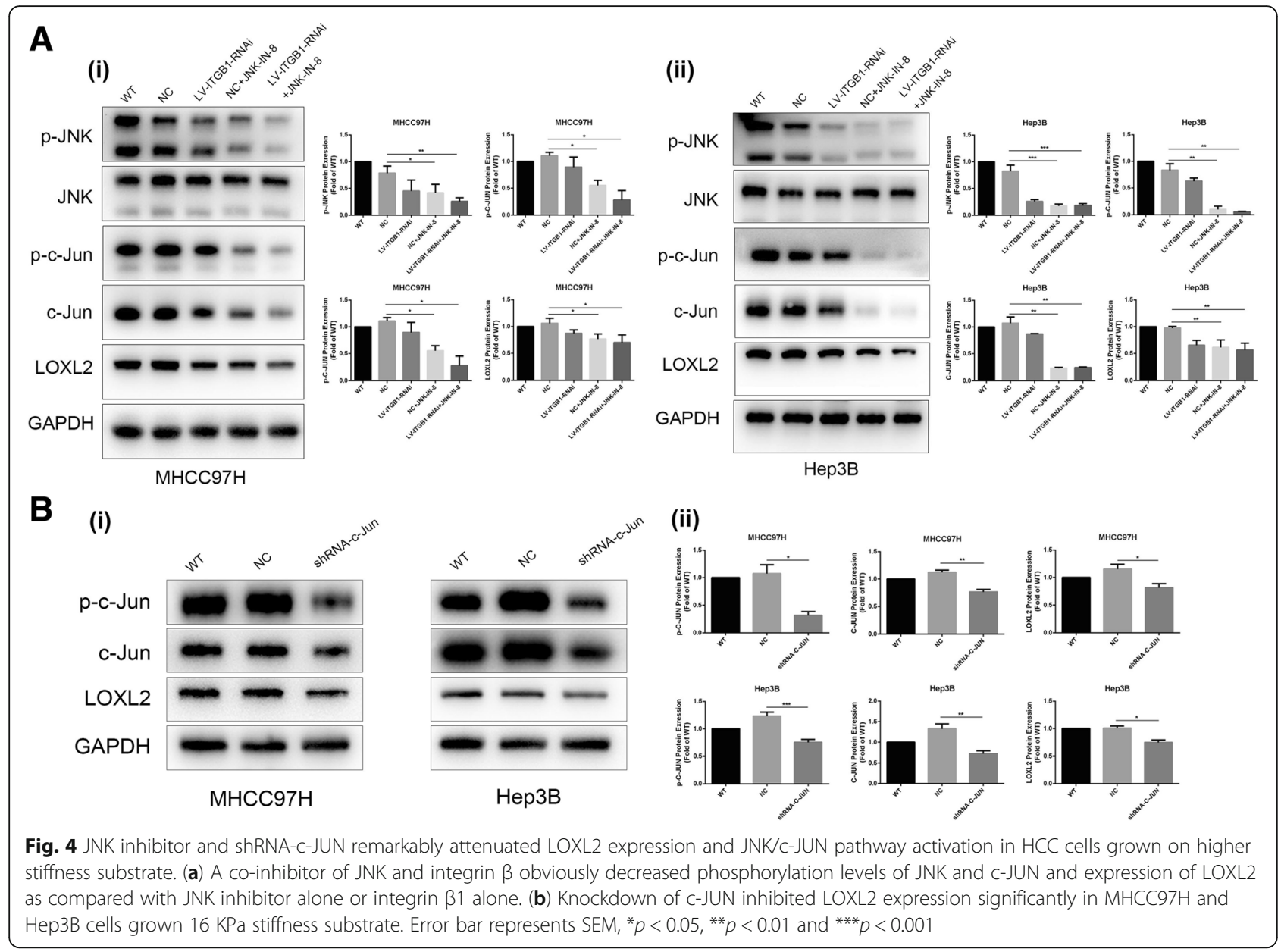

the extracellular matrix that can bind to integrins [29], and plays a crucial role in cell adhesion, growth, migration and differentiation. Our data showed that the number of HCC cells adhered to surface of lung fibroblasts treated with CM-LV-LOXL2-OE was significantly higher than that of the control cells (Fig. 5d), defining the roles of LOXL2 in promoting HCC cells settlement on pre-metastatic niche. Except the above results, we also found that CM-LVLOXL2-OE as chemoattractant increased the number of migrated HCC cells (Fig. 5e), and improve CXCL12 expression in lung fibroblasts (Fig. 5f), revealing that the secreted LOXL2 also facilitated the motility of HCC cells and strengthened CTCs settlement on the remodeled matrix "soil". Accordingly, the secreted LOXL2 form HCC facilitates the formation of pre-metastatic niche and the accomplishment of tumor distant metastasis.

\section{Discussion}

Although LOX family members increase extracellular matrix stiffness and accelerate tumor progression [22, 30, 31 , the effects of matrix stiffness on LOX family member expression are not yet understood. LOX catalyzes the cross-linking of collagen and elastin for maintaining the rigidity and structural stability of the extracellular matrix (ECM) [32, 33]. Overexpressed LOX in cancers is related to tumorigenesis, tumor progression and metastasis [21, 34-37]. LOX and LOXL4 participate in tumor microenvironment remodeling and the formation of premetastatic niches [38, 39]. LOXL2 has similar biological activities in ECM remodeling as LOX, and its overexpression indicates poor prognosis in patients with colon and esophageal squamous cell carcinoma [40], as well as gastric cancer and breast cancer [41, 42]. Highly invasive breast cancer cells instead of non-metastatic cells present obviously high expression of LOXL2 [43, 44]. LOXL2 may be a promising therapeutic target for preventing invasive/ metastatic of breast cancer [41]. LOX and LOXL2 as primary tumor-derived soluble factors contribute to formation of pre-metastatic niches in distant target organs [22, $35,39,45]$. However, little is known about the significance of matrix stiffness-upregulated LOXL2 in pre-metastatic niches formation of HCC.

Higher liver stiffness, as a common physical attribute of chronical liver disease, always accompanies with HCC development, and it is also involved in the regulation of HCC invasion and metastasis $[12,46]$. Wong et 
A
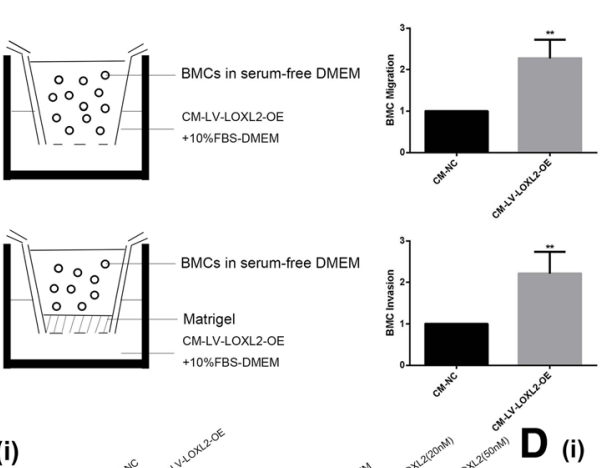

B (i)
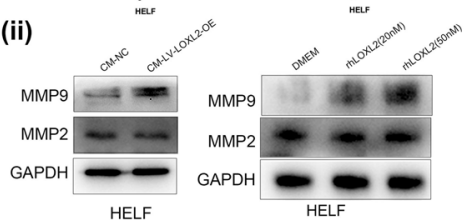

CM-NC

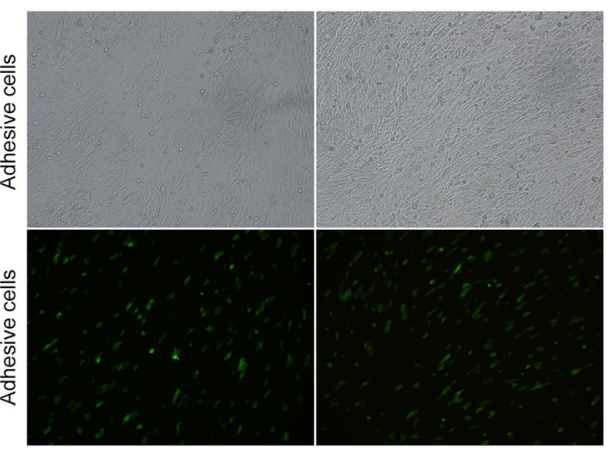

(ii)

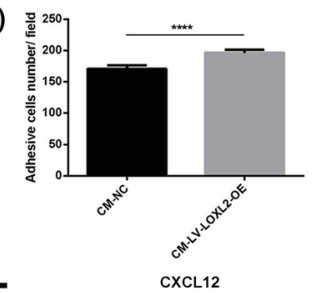

$\mathbf{F}$

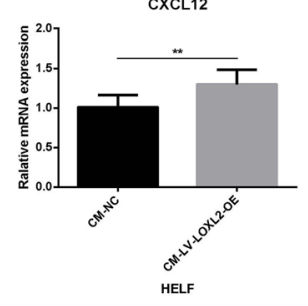

Fig. 5 LOXL2 increases motility and invasion of BMCs, simultaneously improves MMP9 and CXCL12 expression, as well as fibronectin production in lung fibroblasts, which assists assists the formation of pre-metastatic niche. (a) CM-LV-LOXL2-OE as a chemoattractant significantly increased BMCs migration and invasion compared to the control. (b) (i, ii) CM-LV-LOXL2-OE or rhLOXL2 all upregulated MMP9 expression in HELF cells, but had no effect on MMP2 expression. (c) (i-iii) CM-LV-LOXL2-OE or rhLOXL2C activated AKT signaling pathway in HELF cells and upregulated fibronectin expression. (d) (i,ii) The number of HCC cells adhered to surface of lung fibroblasts treated with CM-LV-LOXL2-OE was significantly higher than that of the control cells. (e) (i,ii) CM-LV-LOXL2-OE as chemoattractant increased the number of migrated HCC cells. (f) CM-LV-LOXL2-OE improved CXCL12 expression in lung fibroblasts. Error bar represents SEM, ${ }^{*} p<0.05,{ }^{* *} p<0.01,{ }^{* * *} p<0.001$ and ${ }^{* * *} p<0.0001$

al. [22] proposes that hypoxic or TGF- $\beta$-induced LOXL2 stiffens tumor tissue, and then facilitates the formation of pre-metastasis niche in HCC. Hereby, we speculated that LOXL2 might mediate matrix stiffness-induced effects on pre-metastatic niche. Unlike previous reports on biochemical signal stimuli, the initiator of stimulation in the study was physical mechanical signal. Higher matrix stiffness stimulation remarkably upregulated the expression of LOX family members except LOXL3 in
HCC cells. Based on the expression levels of matrix stiffness-induced LOX members and their potential significance in pre-metastatic niche, we determined to explore underlying molecular mechanism of higher matrix stiffness-induced LOXL2 upregulation, and analyze its significance in pre-metastatic niche formation of HCC. Several literatures have reported that JNK/C-JUN pathway takes part in the regulation of LOXL2 expression [26, 27]. Our results demonstrated that high matrix stiffness activated 
the integrin/JNK/c-JUN signaling pathway and increased LOXL2 expression in HCC cells. Except that, JNK inhibitor partially suppressed phosphorylation levels of JNK and cJUN, and the expression of LOXL2 in HCC cells grown on higher stiffness substrate. ShRNA-c-JUN also downregulated LOXL2 expression significantly. Accordingly, there may be two regulating pathways for higher matrix stiffnessupregulated LOXL2 expression. One way is that increased matrix stiffness activates the phosphorylation JNK and cJUN via integrin $\beta 1 / \alpha 5$, and result in high expression of LOXL2. The other is that higher matrix stiffness directly influences the expression of c-JUN through integrin $\beta 1 / \alpha 5$ and then regulate LOXL2. Although integrin/JNK/c-JUN pathway contributes to higher matrix stiffness-upregulated LOXL2 in HCC cells, we are still unable to exclude the activation of other signal pathways in this regulating process. Inhibition of integrin $\beta 1$ and $\alpha 5$ partially attenuated phosphorylation level of JNK and c-JUN in HCC cell under higher stiffness stimulation (Fig. 3), suggesting that other pathways may participate in matrix stiffness-upregulated LOXL2 in HCC cells. Additionally, the results in the study also implied indirectly that $\beta 1$ integrin family-dependent signaling may determine the functional role of JNK in cancer cells [47].

BMDCs recruitment and matrix remodeling are two common pathological changes in metastatic target organ during the formation of tumor pre-metastatic niche. TDSFs float to distant tissues with blood circulation and promote BMDCs recruitment. The recruited BMDCs interact with resident stromal cells in target organ and produce various growth factors, integrins, chemokines, inflammatory mediators, pro-angiogenic molecules, remodeling matrix microenvironment in target tissue, to facilitate tumor cells colonization and proliferation [48, 49]. In the study, LOXL2 in CM as chemoattractant increased the number of migrated BMCs or invaded BMCs, indicating a significant role of LOXL2 in BMCs recruitment. Additionally, CM-LV-LOXL2-OE and rhLOXL2 also elevated MMP9 expression and fibronectin production significantly in lung fibroblasts, and activation of Akt pathway might participate in LOXL2-induced fibronectin upregulation. Both of MMP9 upregulation and matrix protein deposition triggered matrix remodeling and created a suitable "soil" in lung tissue for circulating tumor cell colonization and growth. Fibronectin is a high-molecular weight glycoprotein of ECM that binds to transmembrane receptor protein integrin, and fibronectin-integrin signaling is closely related to ECM remodeling [50]. Fibronectin implicates on cell adhesion, growth, migration and differentiation [29], as well as carcinoma development [51]. Additionally, fibronectin exerts a crucial role in pre-metastatic niche formation by generating a suitable extracellular matrix "soil" and promoting CTCs settlement on them. TDSFs released by primary tumor promote HPCs recruitment at distant target organ, and recruited HPCs localize to areas of increased fibronectin which is newly synthesized by resident fibroblasts in distant organ. The HPCs, along with fibronectin and associated stromal cells, alter the local microenvironment, leading to the activation of other integrins and the secretion of chemokines such as CXCL12 that promote attachment, survival and growth of tumor cells [52, 53]. Our data revealed that the number of HCC cells adhered to surface of the treated lung fibroblasts with CM-LV-LOXL2OE was obviously increased compared with that of the control cells, defining the roles of LOXL2 in promoting HCC cells settlement on pre-metastatic niche. Besides, CM-LV-

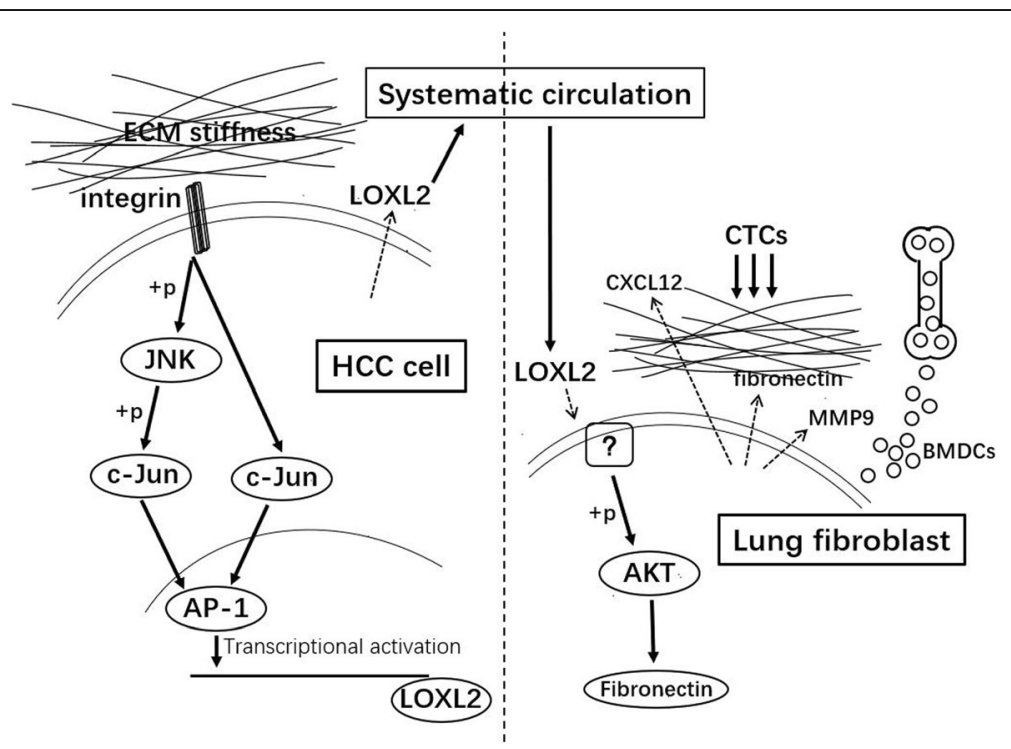

Fig. 6 The proposed mechanism by which higher matrix stiffness upregulates LOXL2 expression in HCC cells, and the secreted LOXL2 promotes fibronectin production, MMP9 and CXCL12 expression and BMDCs recruitment to assist pre-metastatic niche formation 
LOXL2-OE as chemoattractant increased the number of migrated HCC cells, and improve the expression of chemokine CXCL12 in lung fibroblasts, suggesting that the secreted LOXL2 also facilitated the motility ability of HCC cells and strengthened CTCs settlement on the remodeled matrix "soil". Although the data in vitro support a significant role of LOXL2 in facilitating formation of premetastatic niche, it is still necessary to further confirm its roles in animal model in the following study.

\section{Conclusion}

Integrin $\beta 1 / \alpha 5 /$ JNK/c-JUN signaling pathway participates in higher matrix stiffness-induced LOXL2 upregulation in HCC cells. The secreted LOXL2 promotes fibronectin production, MMP9 and CXCL12 expression and BMDCs recruitment to assist pre-metastatic niche formation (Fig. 6).

\section{Additional files}

Additional file 1: Sequence of human LOXL2 overexpression. (DOCX $16 \mathrm{~kb}$ )

Additional file 2: Figure S1. Assessment of transfection efficiency. (A) (i) The transfected HCC cells with LV-LOXL2-OE. (ii) The expression level of LOXL2 in MHCC97H cells transfected with LV-LOXL2-OE. (B) The expression level of integrin $\beta 1$ in HCC cells transfected with LV-ITGB1RNAi. (C) The expression level of integrin a5 in HCC cells transfected with LV-ITGA5-RNAi. (JPG 336 kb)

\section{Abbreviations}

AP-1: Activator protein 1; CM: Conditioned medium; COL1: Collagen 1; CTC: Circulating tumor cell; CXCL12: C-X-C motif chemokine ligand 12; ECM: Extracellular matrixi; GAPDH: Glyceraldehyde-3-phosphate dehydrogenase; HCC: Hepatocellular carcinoma; HELF: Human embryo lung fibroblast; HPC: Haematopoietic progenitor cell; JNK: c-Jun N-terminal kinase; LOX: Lysyl oxidase; LOXL1: Lysyl oxidase like 1; LOXL2: Lysyl oxidase like 2; LOXL3: Lysyl oxidase like 3; LOXL4: Lysyl oxidase like 4; MMP2: Matrix metalloproteinnase 2; MMP9: Matrix metalloproteinase 9; NC: Negative control; OPN: Osteopontin; qRT-PCR: Quantitative reverse transcription polymerase chain reaction; rhLOXL2: Recombinant human Lysyl oxidase like 2; TDSF: Tumor derived soluble factor; TGF- $\beta$ : Transforming growth factor beta; VEGF: Vascular endothelial growth factor

\section{Funding}

This study was sponsored by grants from the National Nature Science Foundation of China $(81573019,81272583)$ and National Youth Natural Science Foundation of China (81401967).

\section{Authors' contributions}

Conceived and designed the experiments: JFC, SFW. Performed the experiments: SFW, QDZ, YYD. Analyzed data: SFW, XXX, YHW. Contributed reagents/materials/analysis tools: $Y Y, R X C, C H, Z M W, J C, D M G, Y Z, T C X, Z G R$. Wrote the paper: SFW, JFC. All authors read and approved the final manuscript.

\section{Ethics approval and consent to participate}

All animal protocols were approved by the Ethical Committee on Animal Experiments of Zhongshan Hospital, Fudan University (Permit Number:2016120).

\section{Competing interests}

The authors declared that they have no competing interests.

\section{Publisher's Note}

Springer Nature remains neutral with regard to jurisdictional claims in published maps and institutional affiliations.

\section{Author details}

${ }^{1}$ Liver Cancer Institute, Zhongshan Hospital, Fudan University and Key Laboratory of Carcinogenesis and Cancer Invasion, Ministry of Education, 136 Yi Xue Yuan Road, Shanghai 200032, People's Republic of China.

${ }^{2}$ Department of Radiology, Shanghai Cancer Center, Fudan University, Shanghai 200032, People's Republic of China. Department of Urology, Zhongshan Hospital, Fudan University, Shanghai 200032, People's Republic of China. ${ }^{4}$ Department of Oncology, Zhongshan Hospital, Fudan University, Shanghai 200032, People's Republic of China.

Received: 19 October 2017 Accepted: 13 April 2018

Published online: 04 May 2018

\section{References}

1. Schrader J, Gordonwalker $\Pi$, Aucott RL, et al. Matrix stiffness modulates proliferation, chemotherapeutic response and dormancy in hepatocellular carcinoma cells. Hepatology. 2011;53(4):1192-205.

2. Swift J, Ivanovska IL, Buxboim A, et al. Nuclear Lamin-a scales with tissue stiffness and enhances matrix-directed differentiation. Science. 2013; 341(6149):975

3. Zhang T, Lin S, Shao X, et al. Effect of matrix stiffness on osteoblast functionalization. Cell Prolif. 2017:50(3):e12338.

4. Wei SC, Laurent F, Tsai JH, et al. Matrix stiffness drives epithelialmesenchymal transition and tumour metastasis through a TWIST1-G3BP2 mechanotransduction pathway. Nat Cell Biol. 2015;17(5):678-88.

5. Tilghman RW, Blais EM, Cowan CR, et al. Matrix rigidity regulates Cancer cell growth by modulating cellular metabolism and protein synthesis. PLoS One. 2012;7(5):e37231.

6. Liu Y, Yan G, Han Y, et al. Tumor Exosomal RNAs promote lung premetastatic niche formation by activating alveolar epithelial TLR3 to recruit neutrophils. Cancer Cell. 2016;30(2):243-56.

7. Le TN, Oscar C, Mouw JK, et al. Loss ofmiR-203regulates cell shape and matrix adhesion through ROBO1/Rac/FAK in response to stiffness. J Cell Biol. 2016;212(6):707-19.

8. Mouw JK, Yui Y, Damiano L, et al. Tissue mechanics modulate microRNAdependent PTEN expression to regulate malignant progression. Nat Med. 2014;20(4):360-7.

9. Tao Z, Tao G, Jing X, et al. Softening substrates promote chondrocytes phenotype via RhoA/ROCK pathway. ACS Appl Mater Interfaces. 2016;8(35): 22884-91.

10. You $Y$, Zheng $Q$, Dong $Y$, et al. Matrix stiffness-mediated effects on stemness characteristics occurring in HCC cells. Oncotarget. 2016;7(22): $32221-31$.

11. Liu C, Liu Y, Xie HG, et al. Role of three-dimensional matrix stiffness in regulating the chemoresistance of hepatocellular carcinoma cells. Biotechnology \& Applied Biochemistry. 2015;62(4):556-62.

12. Zhao G, Cui J, Qin Q, et al. Mechanical stiffness of liver tissues in relation to integrin $\beta 1$ expression may influence the development of hepatic cirrhosis and hepatocellular carcinoma. J Surg Oncol. 2010;102(5):482-9.

13. Parkin DM, Bray F, Ferlay J, et al. Estimating the world cancer burden: Globocan 2000. Int J Cancer. 2001;94(2):153-6.

14. Fattovich $\mathrm{G}$, Stroffolini T, Zagni I, et al. Hepatocellular carcinoma in cirrhosis: incidence and risk factors. Gastroenterology. 2004;127(1):35-50.

15. Masuzaki $\mathrm{R}$, Tateishi $\mathrm{R}$, Yoshida $\mathrm{H}$, et al. Prospective risk assessment for hepatocellular carcinoma development in patients with chronic hepatitis C by transient elastography. J Hepatol. 2009;50(6):1954-61.

16. Dong $Y$, Xie $X$, Wang $Z$, et al. Increasing matrix stiffness upregulates vascular endothelial growth factor expression in hepatocellular carcinoma cells mediated by integrin $\beta 1$. Biochemical \& Biophysical Research Communications. 2014;444(3):427-32.

17. Yang $Y$, Zheng $Q$, Dong $Y$, et al. Higher matrix stiffness upregulates Osteopontin expression in hepatocellular carcinoma cells mediated by integrin $\beta 1 / G S K 3 \beta / \beta$-catenin signaling pathway. PLoS One. 2015;10(8): e0134243.

18. Yang $L$, Cao X. Characteristics and significance of the pre-metastatic niche. Cancer Cell. 2016;30(5):668-81. 
19. Sceneay J, Smyth MJ, Möller A. The pre-metastatic niche: finding common ground. Cancer \& Metastasis Reviews. 2013;32(3-4):449-64.

20. Wang TH, Hsia SM, Shieh TM. Lysyl oxidase and the tumor microenvironment. Int J Mol Sci. 2017;18(1):62

21. Perryman L, Erler JT. Lysyl oxidase in cancer research. Future Oncol. 2014; 10(9):1709-17.

22. Wong CC, Tse AP, Huang Y, et al. Lysyl oxidase-like 2 is critical to tumor microenvironment and metastatic niche formation in hepatocellular carcinoma. Hepatology. 2014;60(5):1645-58.

23. Tey SK, Mao X, Yam JWP. Role of nuclear met-derived exosomes in hepatocellular carcinoma metastasis and lung premetastatic niche formation. Eur J Cancer. 2016;61:S63.

24. Jung PH, Galina G, Wang Z, et al. Deregulation of FoxM1b leads to tumour metastasis. Embo Molecular Medicine. 2011;3(1):21-34.

25. Peinado H, Lavotshkin S, Lyden D. The secreted factors responsible for premetastatic niche formation: old sayings and new thoughts. Semin Cancer Biol. 2011;21(2):139-46.

26. Sethi A, Mao W, Wordinger RJ, et al. Transforming growth factor-beta induces extracellular matrix protein cross-linking lysyl oxidase (LOX) genes in human trabecular meshwork cells. Invest Ophthalmol Vis Sci. 2011;52(8):5240-50.

27. Adamopoulos C, Piperi C, Gargalionis AN, et al. Advanced glycation end products upregulate lysyl oxidase and endothelin-1 in human aortic endothelial cells via parallel activation of ERK1/2-NF-KB and JNK-AP-1 signaling pathways. Cellular \& Molecular Life Sciences Cmls. 2016;73(8):1685-98.

28. Zhang $T$, Inestavaquera $F$, Niepel $M$, et al. Discovery of potent and selective covalent inhibitors of JNK. Chem Biol. 2012;19(1):140-54.

29. Pankov R, Yamada KM. Fibronectin at a glance. J Cell Sci. 2002;115(20):3861-3.

30. Baker AM, Bird D, Lang G, et al. Lysyl oxidase enzymatic function increases stiffness to drive colorectal cancer progression through FAK. Oncogene. 2013;32(14):1863-8.

31. Deng J, Liu Y, Lee $H$, et al. S1PR1-STAT3 signaling is crucial for myeloid cell colonization at future metastatic sites. Cancer Cell. 2012;21(5):642-54.

32. Harris ED. Copper-induced activation of aortic lysyl oxidase in vivo. Proc Natl Acad Sci U S A. 1976;73(2):371-4

33. Piez KA. Cross-linking of collagen and elastin. Annu Rev Biochem. 2003; 37(37):547-70

34. Kasashima $H$, Yashiro $M$, Kinoshita $H$, et al. Lysyl oxidase is associated with the epithelial-mesenchymal transition of gastric cancer cells in hypoxia. Gastric Cancer. 2016;19(2):431-42.

35. Cox TR, Gartland A, Erler JT. Lysyl oxidase, a targetable secreted molecule involved in Cancer metastasis. Cancer Res. 2016;76(2):188-92.

36. Shih YH, Chang KW, Chen MY, et al. Lysyl oxidase and enhancement of cell proliferation and angiogenesis in oral squamous cell carcinoma. Head \& Neck. 2013;35(2):250-6.

37. Osawa T, Ohga N, Akiyama K, et al. Lysyl oxidase secreted by tumour endothelial cells promotes angiogenesis and metastasis. Br J Cancer. 2013; 109(8):2237-47.

38. Wong $\mathrm{CL}$, Gilkes DM, Zhang $\mathrm{H}$, et al. Hypoxia-inducible factor 1 is a master regulator of breast cancer metastatic niche formation. Proc Natl Acad Sci U S A. 2011;108(39):16369-74

39. Erler JT, Bennewith $\mathrm{KL}$, Cox TR, et al. Hypoxia-induced lysyl oxidase is a critical mediator of bone marrow cell recruitment to form the premetastatic niche. Cancer Cell. 2009;15(1):35-44.

40. Fong S, Dietzsch EK, Hollosi P, et al. Lysyl oxidase-like 2 expression is increased in colon and esophageal tumors and associated with less differentiated colon tumors. Genes Chromosomes \& Cancer. 2007;46(7):644-55.

41. Peng L, Ran YL, Hu H, et al. Secreted LOXL2 is a novel therapeutic target that promotes gastric cancer metastasis via the Src/FAK pathway. Carcinogenesis. 2009;30(10):1660-9.

42. Akiri G, Sabo E, Dafni H, et al. Lysyl oxidase-related protein-1 promotes tumor fibrosis and tumor progression in vivo. Cancer Res. 2003;63(7):1657-66.

43. Peinado H, Del Carmen Iglesias-de la Cruz M, Olmeda D, et al. A molecular role for lysyl oxidase-like 2 enzyme in snail regulation and tumor progression. EMBO J. 2005;24(19):3446-58.

44. Kirschmann DA, Seftor EA, Fong SF, et al. A molecular, role, for lysyl, oxidase, in breast, cancer, invasion. Cancer Res. 2002;62(15):4478-83.

45. Canesin G, Cuevas EP, Santos V, et al. Lysyl oxidase-like 2 (LOXL2) and E47 EMT factor: novel partners in E-cadherin repression and early metastasis colonization. Oncogene. 2015;34(8):951-64.

46. Papatheodoridis GV, Idilman R, Dalekos GN, et al. The risk of hepatocellular carcinoma is decreasing after the first 5 years of entecavir or tenofovir in Caucasians with chronic hepatitis B. Hepatology. 2017;66(5):1444-53.

47. Snider $J$, Allison C, Bellaire BH, et al. The $\beta 1$ integrin activates JNK independent of CagA, and JNK activation is required for helicobacter pylori CagA+-induced motility of gastric Cancer cells. J Biol Chem. 2008;283(20):13952-63.

48. Chioda M, Peranzoni E, Desantis G, Papalini F, Falisi E, Solito S, Mandruzzato S, Bronte V. Myeloid cell diversification and complexity: an old concept with new turns in oncology. Cancer Metastasis Rev. 2011;30:27-43.

49. Psaila B, Lyden D. The metastatic niche: adapting the foreign soil. Nat Rev Cancer. 2009;9:285-93.

50. Larsen M, Artym W, Green JA, et al. The matrix reorganized: extracellular matrix remodeling and integrin signaling. Curr Opin Cell Biol. 2006;18(5):463-71.

51. Han S, Khuri FR, Roman J. Fibronectin stimulates non-small cell lung carcinoma cell growth through activation of Akt/mammalian target of rapamycin/S6 kinase and inactivation of LKB1/AMP-activated protein kinase signal pathways. Cancer Res. 2006;66(1):315-23.

52. Kaplan RN, Rafii S, Lyden D. Preparing the "soil": the premetastatic niche. Cancer Res. 2006;66(23):11089-93.

53. Kaplan RN, Riba RD, Zacharoulis S, et al. VEGFR1-positive haematopoietic bone marrow progenitors initiate the pre-metastatic niche. Nature. 2005; 438(7069):820-7.

\section{Ready to submit your research? Choose BMC and benefit from:}

- fast, convenient online submission

- thorough peer review by experienced researchers in your field

- rapid publication on acceptance

- support for research data, including large and complex data types

- gold Open Access which fosters wider collaboration and increased citations

- maximum visibility for your research: over $100 \mathrm{M}$ website views per year

At BMC, research is always in progress.

Learn more biomedcentral.com/submissions 